\title{
Comparison of the association of mammographic density and clinical factors with ductal carcinoma in situ versus invasive ductal breast cancer in Korean women
}

Hyeonyoung Ko ${ }^{1}$, Jinyoung Shin ${ }^{5}$, Jeong Eon Lee², Seok Jin Nam², Tuong Linh Nguyen ${ }^{3}$, John Llewelyn Hopper ${ }^{3,4}$ and Yun-Mi Song ${ }^{1 *}$

\begin{abstract}
Background: In spite of the increasing incidence of in situ breast cancer, the information about the risk factors of in situ breast cancer (DCIS) is scarce as compared to the information available for invasive ductal breast cancer (IDC) , with inconsistent findings regarding the difference in risk factors between DCIS and IDC.

Methods: We enrolled 472 women with IDC and 90 women with DCIS and 1088 controls matching for age and menopausal status. Information on risk factors was collected through self-administered questionnaire. Percent mammographic dense area (PDA), absolute mammographic dense area (ADA), and nondense area were assessed using a computer-assisted thresholding technique. Odds ratio (OR) and 95\% confidence intervals (CI) were estimated by conditional logistic regression model with adjustment for covariates.
\end{abstract}

Results: Later age at menarche and regular physical exercise were associated with decreased risk of IDC, whereas alcohol consumption, previous benign breast disease, and family history of breast cancer were associated with increased risk of IDC. For DCIS, previous benign breast disease and alcohol consumption were associated with the increased risk, and regular physical exercise was associated with decreased risk. Increase of ADA by 1-quartile level and PDA increase by $10 \%$ were associated with $1.10(95 \% \mathrm{Cl}: 1.01,1.21)$ and $1.10(95 \% \mathrm{Cl}: 1.01,1.19)$ times greater risk of IDC, respectively. The increase of ADA by 1-quartile level and PDA increase by 10\% were associated with 1.17 (95\% Cl: $0.91,1.50)$ times and $1.11(95 \% \mathrm{Cl}: 0.90,1.37)$ times greater risk of DCIS, respectively, but the associations were not statistically significant. There was no significant difference in the association with risk factors and mammographic density measures between IDC and DCIS $(P>0.1)$.

Conclusions: Differential associations of DCIS with mammographic density and risk factors as compared with the associations of IDC were not evident. This finding suggests that IDC and DCIS develop through the shared causal pathways.

Keywords: Mammographic density, Ductal carcinoma in situ, Invasive ductal breast cancer

\footnotetext{
* Correspondence: yunmisong@skku.edu

Hyeonyoung Ko and Jin-Young Shin are joint first authors.

'Department of Family Medicine, Samsung Medical Center, Sungkyunkwan

University School of Medicine, 81 Irwon-ro, Gangnam-gu, Seoul 135-710,

South Korea

Full list of author information is available at the end of the article
} 


\section{Background}

Over the past several decades, the incidence rate of in situ breast cancer has increased worldwide, probably due to the widespread use of mammograms for breast cancer screening [1-3].

Ductal carcinoma in situ (DCIS), the most common type of in situ breast cancer, is the proliferation of presumably malignant epithelial cells confined to the mammary ducts and lobules without evident stromal invasion through the basement membrane [4]. DCIS is considered as a precursor lesion of invasive ductal cancer (IDC) in the middle of progressive changes in nuclear features from normal breast tissue to invasive breast cancer [5]. Approximately four-fold higher risk of IDC was reported in women diagnosed with DCIS [6]. Longterm studies on women with DCIS treated by diagnostic biopsy alone revealed that not all but substantially large proportion of the women were diagnosed with IDC over the course of follow up [7]. Expression of biological markers such as estrogen receptor (ER), progesterone receptor (PgR), and human epidermal growth factor receptor-2 (HER2) was found to be similar between in situ component and invasive component in breast samples with both DCIS and IDC $[8,9]$. In addition, the same tumor suppressor gene in chromosome 11 was mutated or missing in both invasive and in situ breast cancer [10], and a study that compared 12 susceptibility loci found no strong evidence of presence of a different association between DCIS and IDC [11].

However, findings regarding the difference in risk factors between DCIS and IDC were inconsistent, and the information about the risk factors of DCIS was less available than for IDC, especially for Asian women. Some studies reported similar associations with risk factors such as family history of breast cancer, previous breast biopsy, or parity between DCIS and IDC [11-14], whereas other studies reported differential association $[15,16]$. Mammographic density (MD) reflects the relative amount of fat, connective tissue, and epithelial tissue in breast. Studies have consistently reported MD as a significant strong risk factor for breast cancer independent of other breast cancer risk factors, for western as well as for Asian women population [17, 18]. However, it is also controversial whether MD differentially affects the risk of developing breast cancer between DCIS and IDC. Yaghjyan L et al. [19] found that in situ breast cancer had a stronger association with MD measured by percent breast density than invasive breast cancer, whereas other reported no differential association with respect to MD between DCIS and IDC [20, 21].

We therefore conducted a case-control study in Korean women to evaluate the associations of breast cancer with risk factors including MD, separately for DCIS and IDC. Considering that most of the previous studies have been conducted on Western population and information on the risk factors of breast cancer by invasiveness for Asian women population was scarce, it is hypothesized that the findings from this study on the extent to which DCIS and IDC share the risk factors may provide awareness regarding the natural history of breast cancer in Asian women.

\section{Methods}

\section{Study design and study subjects}

We included a total of 562 breast cancer patients (472 IDC and 90 DCIS), who received curative surgery at Samsung Medical Center between February 2006 and August 2013 and had available data for MD and pathologic status. Of the 562 cases, 186 cases were recruited retrospectively from the Health Promotion Center of the Samsung Medical Center, while 376 cases were prospectively recruited from the department of surgery. Controls were randomly selected from 6863 women who had repeatedly (at least three times) received a periodic health checkup at the Health Promotion Center in the Samsung Medical Center and had no evidence of malignant breast disease for at least 1 year after the time at which mammogram for the present study was taken. We selected two controls for each breast cancer case through individual matching for menopausal status and age (within one year) at which mammogram was taken, except for 36 cases for whom only one control could be selected because of the limited control pool within matching strata. Thus, 1088 controls (912 for IDC cases and 176 for DCIS cases) were included in the final analysis. This study was approved by the Institutional Review Board of Samsung Medical Center (SMC 201106-052-022). The Board waived informed consent for the retrospectively recruited subjects, and all prospectively recruited subjects provided written informed consent.

\section{Mammographic density measurements}

Mammograms were taken at the same institution using a full-field digital mammography system such as Senograph 2000D/DMR/DS (General Electric Company, Milwaukee, WI, USA) or Selenia (Hologic, Inc. Bedford, MA, USA). For breast cancer cases, MD of the breast contralateral to the breast involved in the cancer diagnosis was measured in the mammograms taken 1.0 (standard deviation: 2.1) months prior to the diagnosis. For controls, MD of the right breast was measured. Single observer who was blinded to all identifying information completed the measurement of total breast area $\left(\mathrm{cm}^{2}\right)$ and area of mammographically dense tissue (ADA, $\mathrm{cm}^{2}$ ) directly from the cranio-caudal view using the computerassisted thresholding technique (Cumulus: Imaging Research Program, Sunnybrook Health Sciences Centre, 
University of Toronto, Toronto, Canada). Subsequently, we calculated nondense area $\left(\mathrm{cm}^{2}\right)$ of breast by subtracting ADA from total breast area and percentage dense area (PDA) as ADA divided by total breast area. MD measurement by Cumulus was reported to be highly reproducible [18]. Estimates of intraclass correlation coefficients for repeatedly measured MD were 0.99 for total breast area and 0.98 for ADA [19]. We categorized total breast area, nondense area, and ADA into four levels based on the quartile distribution of mammographic measures in the control group. PDA was categorized into five levels by $10 \%$ interval.

\section{Other measurements}

We obtained information about pathological examinations and hormone receptor status by reviewing electronic medical records of the breast cancer cases. Expression of ER, PgR, and HER2 was assessed by immunohistochemistry staining kits: ER by 6F11 (Novocastra Laboratories, Newcastle upon Tyne, UK), PgR by IA6 (Novocastra Laboratories, Newcastle upon Tyne, UK), and HER2 by CB11 (Novocastra Laboratories, Newcastle upon Tyne, UK). ER and PgR positivity was defined as an Allred score of 3 to 8 . Allred scoring semi-quantitatively measures the proportion of positive cells on 0 to 5 scales and staining intensity on a 0 to 3 scale. Positivity for HER2 overexpression was defined as a score of $3+$ (strong, complete membrane immunoreactivity in $>10 \%$ of tumor cells) on immunohistochemistry or as a gene amplification ratio $\geq 2.0$ by fluorescence in situ hybridization using PathVysion HER2 DNA Probe kits (Abbott Molecular Inc., Des Plaines, IL, USA).

Family history of breast cancer among first-degree relatives (mother, daughter, or sister), previous benign breast disease; menstrual and reproductive history (age at menarche, menopausal status, use of estrogen replacement therapy, and number of live birth); and health-related behaviors (smoking, alcohol consumption, and physical activity) were collected using a selfadministered questionnaire. All control subjects and retrospectively recruited 186 cases completed the questionnaire on the same day when they received a mammogram, and prospectively recruited cases completed the questionnaire when they were admitted to the hospital for surgical treatment. We defined a woman postmenopausal if she had no menstrual period for at least one year, has ever received hormone replacement therapy, or aged over 55 years. Study participants were divided into two or three categories for each of the following variables: alcohol consumption (ever, never), smoking (ever, never), frequency of regular physical exercise ( $\geq 1$ /week, $<1$ / week), and use of hormone replacement therapy (ever, never). Body mass index (BMI, $\mathrm{kg} / \mathrm{m}^{2}$ ) was calculated using measured height $(\mathrm{cm})$ and weight $(\mathrm{kg})$.

\section{Statistical analysis}

For some variables with missing data (i.e., age at menarche), we imputed data using the average value for the variable among controls in the same age group. We compared demographic and clinical characteristics between cases and controls by paired $t$ test and Cochran-MantelHaenszel chi-square test. We also compared demographic and clinical characteristics between IDC and DCIS cases by $t$ test and chi-square test.

We estimated odds ratio (OR) with $95 \%$ confidence intervals $(95 \% \mathrm{CI})$ for DCIS and IDC associated with MD and clinical risk factors by fitting a conditional logistic regression model for matched case-control study data. For estimating the association between MD and breast cancer, we adjusted covariates including age, menopausal status, height, BMI, age at menarche, number of live birth, smoking status, alcohol consumption, regular physical exercise, family history of breast cancer, previous benign breast disease, and use of estrogen replacement therapy. Furthermore, to reduce he probable confounding by the different recruitment method, we adjusted the recruitment method in addition.

We evaluated whether the association of breast cancer and clinical risk factors with MD differs between IDC and DCIS by adding interaction terms (invasiveness $\mathrm{x}$ each variable) to the analytic model.

In addition, we did stratified analysis according to the method of case recruitment and checked whether there is influence of recruitment method by examining interactions between the recruitment center and the variables on the breast cancer risk, separately for DCIS and IDC. We also did stratified analysis to examine whether the association of BMI with DCIS and IDC differed according to the menopausal status, and checked interaction between menopausal status and BMI, separately for DCIS and IDC.

All statistical analyses used the SAS statistical package (SAS Institute, Cary, NC, USA) with the level of statistical significance set as $P=0.05$.

\section{Results}

DCIS cases occupied 16.0\% (90 cases) of all the breast cancer cases. Clinical and lifestyle characteristics and mammographic measures were compared between cases and controls, and between IDC and DCIS (Table 1). There was significant difference in BMI, age at menarche, number of live birth, use of estrogen replacement, alcohol consumption, smoking, physical exercise, previous benign breast disease, family history of breast cancer, and all MD measures between breast cancer cases and controls. Compared to IDC, DCIS cases had lower mean BMI and were less likely to be involved in frequent ( $\geq 3$ /week) regular physical exercise. Although IDC cases had greater total and nondense breast area, ADA and 
Table 1 Comparisons of demographic and clinical characteristics between cases and controls and between invasive ductal carcinoma and ductal carcinoma in situ

\begin{tabular}{|c|c|c|c|c|c|c|}
\hline Variables & $\begin{array}{l}\text { All cases } \\
(n=562)\end{array}$ & $\begin{array}{l}\text { Invasive Ductal } \\
\text { carcinoma }(n=472)\end{array}$ & $\begin{array}{l}\text { Ductal carcinoma } \\
\text { in situ }(n=90)\end{array}$ & $\begin{array}{l}\text { Control } \\
(n=1088)\end{array}$ & $P_{\text {difference* }}$ & $P_{\text {difference }} \dagger$ \\
\hline Age at mammogram, years, mean (SD) & $48.7(7.5)$ & $48.9(7.6)$ & $48.1(7.1)$ & $49.3(7.1)$ & $<0.001$ & 0.358 \\
\hline Premenopausal, N (\%) & $389(69.2)$ & $323(68.4)$ & $66(73.3)$ & $742(65.6)$ & - & 0.356 \\
\hline Body mass index, $\mathrm{kg} / \mathrm{m}^{2}$, mean (SD) & $22.9(2.9)$ & $23.1(3.0)$ & $21.8(2.4)$ & $22.5(2.8)$ & 0.002 & $<0.001$ \\
\hline Age at menarche, years, mean (SD) & $14.7(1.7)$ & $14.6(1.7)$ & $14.8(1.7)$ & $14.9(1.6)$ & 0.003 & 0.357 \\
\hline Number of live birth, mean (SD) & $1.9(0.9)$ & $1.9(0.9)$ & $1.8(1.0)$ & 2.0(1.0) & 0.001 & 0.714 \\
\hline Ever-use of estrogen replacement, N (\%) & $40(7.1)$ & $36(7.6)$ & $4(4.4)$ & $107(9.8)$ & 0.066 & 0.489 \\
\hline Ever alcohol consumption, N (\%) & $271(48.2)$ & $223(47.3)$ & $48(53.3)$ & $413(38.0)$ & $<0.001$ & 0.290 \\
\hline Ever smoking, N (\%) & $46(8.2)$ & $40(8.5)$ & $6(6.7)$ & $54(5.0)$ & 0.009 & 0.566 \\
\hline Doing physical exercise $\geq 3 /$ week, N (\%) & $185(32.9)$ & $164(34.8)$ & $21(23.3)$ & $280(25.8)$ & 0.001 & 0.035 \\
\hline Doing physical exercise, $\geq 1 /$ week, N (\%) & 299(53.2) & $248(52.5)$ & $51(56.7)$ & $902(82.9)$ & $<0.001$ & 0.472 \\
\hline Previous benign breast disease, N (\%) & $155(27.6)$ & $127(26.9)$ & $28(31.1)$ & $71(6.5)$ & $<0.001$ & 0.413 \\
\hline Breast cancer among first degree relatives, N (\%) & $56(10.0)$ & $49(10.4)$ & $7(7.8)$ & $42(3.9)$ & $<0.001$ & 0.450 \\
\hline \multicolumn{7}{|l|}{ Tumor marker status, N (\%) } \\
\hline Estrogen receptor $(+)$ & $444(79.1)$ & $373(79.0)$ & $71(79.8)$ & & & 0.873 \\
\hline Progesterone receptor $(+)$ & $401(71.5)$ & $334(70.8)$ & $67(75.3)$ & & & 0.387 \\
\hline Human epidermal growth factor receptor-2 (+) & $134(25.2)$ & $103(23.0)$ & $31(36.5)$ & & & 0.009 \\
\hline \multicolumn{7}{|l|}{ Mammographic density measures } \\
\hline Total breast area, $\mathrm{cm}^{2}$, mean (SD) & 101.9(37.2) & $103.9(38.0)$ & $91.3(31.1)$ & $94.9(33.6)$ & $<0.001$ & 0.001 \\
\hline Dense area, $\mathrm{cm}^{2}$, mean (SD) & $20.4(14.5)$ & $20.7(14.8)$ & 19.1(13.0) & $17.0(12.6)$ & $<0.001$ & 0.355 \\
\hline Nondense area, $\mathrm{cm}^{2}$, mean (SD) & $81.5(36.3)$ & $83.2(36.6)$ & $72.1(33.2)$ & $78.0(33.5)$ & 0.033 & 0.008 \\
\hline Percent density, \%, mean (SD) & $21.4(13.5)$ & $21.1(13.2)$ & $22.8(14.9)$ & 18.8(12.2) & $<0.001$ & 0.287 \\
\hline
\end{tabular}

$S D$ standard deviation, $N$ number

*P value for the difference between cases and matched controls was assessed by paired t test for continuous variables or Cochran-Mantel-Haenszel chi-square test for categorical variables

$\dagger P$ value for the difference between the cases with invasive ductal carcinoma and the cases with ductal carcinoma in situ was assessed by student $t$ test for continuous variables or Chi-square test for categorical variables

PDA did not differ between IDC and DCIS cases. There was no difference in ER and PgR positive status between IDC and DCIS cases, whereas DCIS cases were more likely to be HER2 positive than IDC cases were.

Table 2 shows the associations of DCIS and IDC with clinical characteristics after adjusting for other variables as compared to the matched controls. Later age at menarche (OR $(95 \% \mathrm{CI}): 0.94(0.88,0.99))$ and regular physical exercise for $\geq 1 /$ week (OR $(95 \% \mathrm{CI})$ were $0.45(0.37$, 0.54) were associated with decreased risk of IDC, whereas alcohol consumption (OR (95\%CI): 1.19(0.99, $1.44)$ ), previous benign breast disease (OR $(95 \% \mathrm{CI}): 2.31$ $(1.86,2.86))$, and history of breast cancer among first degree relatives (OR $(95 \% \mathrm{CI}): 1.43(1.05,1.95))$ were associated with increased risk of IDC. For DCIS, alcohol consumption (OR (95\% CI): $1.81(1.14,2.89))$ and previous history of benign breast disease (OR (95\%CI): 2.04 $(1.23,3.39))$ showed a significantly increased risk. Regular physical exercise for $\geq 1$ /week (OR (95\%CI): 0.52(0.31, $0.87)$ ) was associated with decreased risk of DCIS. When we examined that the associations between candidate risk factors and breast cancer were modified by pathologic type of invasiveness, there was no significant difference in the association between IDC and DCIS $(P>0.1)$.

We checked the influence by the method of case recruitment (Additional file 1: Table S1). A significant interaction by the method of case recruitment was found on the association of IDC with ever-use of estrogen replacement and regular physical exercise, with different direction of association. Although there was a significant interaction between recruitment method and the history of previous benign disease on the risk of DCIS, the direction of the association was same with much higher OR in prospectively recruited subjects than retrospectively recruited subjects.

Table 3 shows the association of IDC and DCIS with each MD measure after adjusting for covariates. Total breast area and nondense area were not associated with the risk of both IDC and DCIS. ADA and PDA were positively associated with the IDC. Increase in ADA by 
Table 2 Multivariable adjusted associations of clinical and reproductive characteristics with invasive ductal carcinoma and ductal carcinoma in situ

\begin{tabular}{|c|c|c|c|c|c|c|c|c|c|}
\hline \multirow[b]{3}{*}{ Variables } & \multicolumn{4}{|c|}{ Invasive ductal cancer } & \multicolumn{4}{|c|}{ Ductal carcinoma in situ } & \multirow{3}{*}{$\begin{array}{l}P^{\ddagger} \text { for } \\
\text { interaction }\end{array}$} \\
\hline & \multicolumn{2}{|c|}{ Measurement* } & \multicolumn{2}{|l|}{ Association } & \multicolumn{2}{|c|}{ Measurement* } & \multicolumn{2}{|l|}{ Association } & \\
\hline & $\begin{array}{l}\text { Cases } \\
(n=472)\end{array}$ & $\begin{array}{l}\text { Controls } \\
(n=912)\end{array}$ & $\overline{\mathrm{OR}(95 \% \mathrm{Cl})^{+}}$ & $P$ value & $\begin{array}{l}\text { Cases } \\
(n=90)\end{array}$ & $\begin{array}{l}\text { Controls } \\
(n=176)\end{array}$ & $\overline{\mathrm{OR}}(95 \% \mathrm{Cl})^{+}$ & $P$ value & \\
\hline ody mass index, increase by $1 \mathrm{~kg} / \mathrm{m}^{2}$ & $23.1(3.0)$ & $22.5(2.8)$ & $1.01(0.98,1.04)$ & 0.581 & $21.8(2.4)$ & $22.1(2.8)$ & $0.94(0.86,1.03)$ & 0.210 & 0.259 \\
\hline Age at menarche, increase by 1 - year & $14.6(1.7)$ & $14.9(1.6)$ & $0.94(0.88,0.99)$ & 0.030 & $14.8(1.7)$ & $14.8(1.5)$ & $1.08(0.92,1.26)$ & 0.370 & 0.320 \\
\hline Number of live birth, increase by 1-person & $1.9(0.9)$ & $2.1(1.0)$ & $0.94(0.85,1.03)$ & 0.180 & $1.8(1.0)$ & $2.0(1.0)$ & $0.89(0.71,1.11)$ & 0.304 & 0.781 \\
\hline Ever-use of estrogen replacement & $36(7.6)$ & $96(10.5)$ & $0.88(0.59,1.30)$ & 0.515 & $4(4.4)$ & $11(6.3)$ & $1.04(0.31,3.41)$ & 0.955 & 0.901 \\
\hline Ever alcohol consumption & 223(47.3) & 349(38.3) & $1.19(0.99,1.44)$ & 0.068 & $48(53.3)$ & $64(36.4)$ & $1.81(1.14,2.89)$ & 0.013 & 0.105 \\
\hline Ever smoking & $40(8.5)$ & $43(4.7)$ & $1.16(0.83,1.63)$ & 0.381 & $6(6.7)$ & $11(6.3)$ & $0.96(0.37,2.53)$ & 0.934 & 0.910 \\
\hline Regular physical exercise( $\geq 1$ /week) & $248(52.5)$ & 754(82.8) & $0.45(0.37,0.54)$ & $<0.001$ & $51(56.7)$ & 148(84.1) & $0.52(0.31,0.87)$ & 0.013 & 0.434 \\
\hline Previous benign breast disease & $49(10.4)$ & $39(4.3)$ & $2.31(1.86,2.86)$ & $<0.001$ & $28(31.1)$ & $16(9.1)$ & $2.04(1.23,3.39)$ & 0.006 & 0.484 \\
\hline Breast cancer among first degree relatives & $127(26.9)$ & $55(6.0)$ & $1.43(1.05,1.95)$ & 0.025 & $7(7.8)$ & $3(1.7)$ & $2.14(0.84,5.44)$ & 0.109 & 0.701 \\
\hline
\end{tabular}

*Presented by mean (standard deviation) for continuous variables or number (\%) for categorical variables

tOdd ratio (OR) and 95\% confidence intervals $(\mathrm{Cl})$ were estimated by conditional logistic regression analysis after adjusting for age, menopausal status, height, body mass index, age at menarche, number of children, ever smoking status, alcohol consumption, regular physical exercise, family history of breast cancer among first degree relatives, past history of benign breast disease, use of estrogen replacement, and the method of recruiting subjects

\#Estimated by putting interaction term (each variable $\mathrm{X}$ invasiveness) in the conditional logistic regression model with adjustment for covariates

1-quartile level was associated with 1.10 (95\% CI: 1.01, 1.21) times greater risk of IDC and 1.17 (95\% CI: 0.91, 1.50) times greater risk of DCIS. Increase in PDA by $10 \%$ was associated with 1.10 (95\% CI: $1.01,1.19)$ times greater risk of IDC and 1.11 (95\% CI: 0.90, 1.37) times greater risk of DCIS. Although the associations between DCIS and ADA, PDA were not statistically significant, there was no difference in the association with MD between IDC and DCIS: the P for interactions by invasiveness of breast cancer was 0.426 and 0.666 for the association of breast cancer with $\mathrm{ADA}$ and PDA, respectively.

\section{Discussion}

In the present case-control study on Korean women, the direction and the size of estimates for the association of DCIS with reproductive factors and MD were similar to those of IDC, and no significant heterogeneity in the association between DCIS and IDC was found.

Mammographic density is a well-established strong risk factor for invasive breast cancer [17]. Epidemiologic studies have revealed significant association between breast in situ cancer and MD [21-23]. Interestingly, some study findings suggested the possibility of existence of stronger association between MD and DCIS than that between MD and IDC. A case study found that most of the DCIS lesions (21 of 22) occurred from areas of dense tissue [24]. In a study of Canadian cohort, the relative risk for detecting breast atypia or DCIS in biopsy specimens from women with more than $75 \%$ density was estimated to be 9.7 times higher when compared with that from women showing no mammographic density [25]. A possible explanation for the probably stronger association of MD with DCIS than that with IDC was that radiographic appearance of in situ cancer might result in higher sensitivity of screening mammography for detection of DCIS as compared with IDC detection [26]. In accordance with this suggestion, in a nested case-control study, the OR $(6.58,95 \% \mathrm{CI}=3.47$, $12.48)$ for in situ breast cancer associated with the highest category of PDA ( $\geq 50 \%$ ) as compared with the lowest PDA $(<10 \%)$ was significantly higher than the OR $(3.00$, 95\% CI: 2.13, 4.23) for invasive breast cancer (P for heterogeneity <0.01) [19]. However, other studies showed that the association of MD did not differ between in situ cancer and invasive cancer. A case only study by Ghosh et al. [27], revealed no difference in the ADA and PDA between IDC, DCIS, invasive lobular cancer, and lobular carcinoma in situ after adjusting for covariates. In a nested case-control study within the multiethnic cohort, for the highest category of PDA $(\geq 50 \%)$ and ADA $\left(\geq 45 \mathrm{~cm}^{2}\right)$ as compared with the lowest $\left(<10 \%,<15 \mathrm{~cm}^{2}\right)$, the ORs were 3.58 (95\% CI: $2.26,5.66)$ and $2.92(95 \% \mathrm{CI}$ : $2.01,4.25)$ for IDC, and 2.86 (95\% CI: 1.38, 5.94) and 2.59 (95\% CI: 1.39, 4.82) for DCIS [20] without statistically significant difference between IDC and DCIS [20]. A large study including 3414 cases and 7199 controls also found $2.21(95 \% \mathrm{CI}: 1.92,2.55)$ and 1.87 (95\% CI: $1.42,2.48)$ times higher risk of IDC and DCIS, respectively for high $(>51 \%)$ versus average $(11-25 \%)$ density group, also without significant heterogeneity [28]. A British case-control study reported that the OR of IDC (1.3) associated with denser breast as compared with less dense breast was similar to the OR of DCIS (1.3) [23]. In 
Table 3 Multivariable adjusted association of mammographic density measures with invasive ductal carcinoma and ductal carcinoma in situ

\begin{tabular}{|c|c|c|c|c|c|c|}
\hline & \multicolumn{3}{|l|}{ Invasive ductal cancer } & \multicolumn{3}{|l|}{ Ductal carcinoma in situ } \\
\hline & Number, Cases/controls & OR $(95 \% \mathrm{Cl})^{*}$ & $\overline{P \text { value }}$ & Number, Cases/controls & OR $(95 \% \mathrm{Cl})^{*}$ & $P$ value \\
\hline \multicolumn{7}{|l|}{$\overline{\text { Total area }\left(\mathrm{cm}^{2}\right)^{\dagger}}$} \\
\hline Q1 (< 71.2985) & $95 / 220$ & 1 & & $23 / 52$ & 1 & \\
\hline Q2 (71.2985-89.9320) & $94 / 225$ & $0.99(0.74,1.33)$ & 0.957 & $23 / 47$ & $1.42(0.75,2.72)$ & 0.285 \\
\hline Q3 (89.9321-111.8983) & $121 / 235$ & $0.98(0.73,1.31)$ & 0.981 & $28 / 37$ & $1.93(0.96,3.90)$ & 0.067 \\
\hline Q4 (111.8984 $\leq)$ & $162 / 232$ & $1.17(0.86,1.58)$ & 0.319 & $16 / 40$ & $1.79(0.78,4.11)$ & 0.171 \\
\hline 1-quartile increase & $472 / 912$ & $1.05(0.95,1.16)$ & 0.323 & $90 / 176$ & $1.24(0.96,1.61)$ & 0.095 \\
\hline \multicolumn{7}{|l|}{ P for Interaction ${ }^{\S}=0.998$} \\
\hline \multicolumn{7}{|l|}{ Absolute dense area $\left(\mathrm{cm}^{2}\right)^{+}$} \\
\hline Q1 (< 8.09656) & $82 / 230$ & 1 & & $19 / 42$ & 1 & \\
\hline Q2 (8.09656-14.46536) & $107 / 217$ & $1.27(0.94,1.72)$ & 0.116 & $14 / 55$ & $0.59(0.27,1.28)$ & 0.184 \\
\hline Q3 (14.46537-22.46433) & $113 / 225$ & $1.23(0.91,1.68)$ & 0.176 & $32 / 47$ & $1.11(0.54,2.27)$ & 0.780 \\
\hline Q4 $(22.46434 \leq)$ & $170 / 240$ & $1.41(1.05,1.90)$ & 0.022 & $25 / 32$ & $1.35(0.64,2.83)$ & 0.434 \\
\hline 1-quartile increase & $472 / 912$ & $1.10(1.01,1.21)$ & 0.039 & $90 / 176$ & $1.17(0.91,1.50)$ & 0.218 \\
\hline \multicolumn{7}{|l|}{$P$ for Interaction ${ }^{\S}=0.426$} \\
\hline \multicolumn{7}{|l|}{ Non-dense area $\left(\mathrm{cm}^{2}\right)^{\dagger}$} \\
\hline Q1 (< 54.8846) & $109 / 219$ & 1 & & $31 / 53$ & 1 & \\
\hline Q2 (54.8846-70.4895) & $92 / 231$ & $0.87(0.65,1.16)$ & 0.330 & $20 / 41$ & $1.41(0.73,2.72)$ & 0.306 \\
\hline Q3 (70.4896-93.2822) & $120 / 236$ & $0.93(0.70,1.24)$ & 0.618 & $22 / 36$ & $1.74(0.84,3.61)$ & 0.134 \\
\hline Q4 $(93.2823 \leq)$ & $151 / 226$ & $1.03(0.75,1.41)$ & 0.849 & $17 / 46$ & $1.48(0.62,3.49)$ & 0.376 \\
\hline 1-quartile increase & $472 / 912$ & $1.02(0.92,1.13)$ & 0.747 & $90 / 176$ & $1.17(0.90,1.53)$ & 0.251 \\
\hline \multicolumn{7}{|l|}{ P for Interaction ${ }^{\S}=0.693$} \\
\hline \multicolumn{7}{|l|}{ Percentage dense area ${ }^{\ddagger}$} \\
\hline$<10 \%$ & $98 / 248$ & 1 & & $21 / 48$ & 1 & \\
\hline 10-19\% & $151 / 285$ & $1.29(0.97,1.70)$ & 0.076 & $17 / 60$ & $0.68(0.33,1.43)$ & 0.310 \\
\hline $20-29 \%$ & $116 / 219$ & $1.37(1.01,1.85)$ & 0.044 & $32 / 42$ & $1.19(0.58,2.45)$ & 0.641 \\
\hline $30-39 \%$ & $62 / 98$ & $1.36(0.95,1.95)$ & 0.095 & $7 / 24$ & $0.55(0.19,1.58)$ & 0.267 \\
\hline$\geq 40 \%$ & $45 / 62$ & $1.54(1.02,2.31)$ & 0.039 & $13 / 2$ & $1.90(0.72,5.06)$ & 0.197 \\
\hline $10 \%$ increase & $472 / 912$ & $1.10(1.01,1.19)$ & 0.029 & $90 / 176$ & $1.11(0.90,1.37)$ & 0.348 \\
\hline P for Interaction ${ }^{\S}=0.666$ & & & & & & \\
\hline
\end{tabular}

*Odd ratio (OR) and 95\% confidence intervals (Cl) were estimated by conditional logistic regression analysis after adjusting for age, menopausal status, height, body mass index, age at menarche, number of children, ever smoking status, alcohol consumption, regular physical exercise, family history of breast cancer among first degree relatives, past history of benign breast disease, use of estrogen replacement, and the method of recruiting subjects

tQuartiles $(\mathrm{Q})$ were determined based on the distribution of mammographic measures of control group. Q1 is the lowest quartile level and Q4 is the highest quartile level

¥Calculated as the dense area divided by total breast area

$\S$ Estimated by putting interaction term (unit of increase in each mammographic density measure $\mathrm{X}$ invasiveness) in the conditional logistic regression model after adjusting for covariates

our study, although the association between MD and DCIS did not reach statistical significance, the risk estimates for the association of DCIS with both ADA and PDA were almost similar with those for IDC, and they did not significantly differ from the risk estimate for IDC, as found in the British study [23].

Interestingly, the estimates (OR (95\% CI) for the association between MD and breast cancer in our study tend to be weaker than the magnitude of association observed in the above mentioned studies in Western population: the risk associated PDA $\geq 40 \%$ was $1.54(1.02,2.31)$ for IDC and $1.90(0.72,5.06)$ for DCIS in our study. Different strength of association between MD and breast cancer has been frequently reported across different ethnic groups $[29,30]$, and the association observed in Asian women tended to be weaker than the association in women from Western populations [31, 32]. In a previous meta-analysis, the relative risk ratio of developing breast 
cancer for women in Wolfe's most-dense category (DY) compared with those in the least-dense category (N1) was 3.98 (95\% CI: $2.53,6.27)$ from an incidence study, and 2.42 (95\% CI: $1.98,2.97)$ from a prevalence study [32]. In comparison, from a Japanese case-control study, the relative risk was 2.20 (95\% CI: 1.02, 4.77) for DY group compared with N1 groups [31]. However, because of the lack of Asian studies on MD and different pathologic type of breast cancer, we could not directly compare the difference in the strengths of association of IDC and DCIS between Western and Asian population.

The association between age at menarche and in situ cancer was controversial with either null $[12,13,16]$ or inverse association [33]. In our study, age at menarche had an inverse association with IDC, but not with DCIS. However, no heterogeneity regarding the association with the age at menarche existed between IDC and DCIS. We assume that this conflicting finding might have been caused by the inadequate sample size of DCIS in our study. However, given that some studies with large enough sample size have reported no association $[12,13]$, the association between age at menarche and DCIS in Asian population needs further evaluation in a study with large enough sample size.

In the present study, we found no significant association of BMI with IDC as well as DCIS. In studies that did not differentiate premenopausal and postmenopausal breast cancer, no association between BMI and in situ cancer and a positive association between BMI and invasive cancer have been reported. $[13,16]$ Most of the previous studies have reported presence of significant inverse association between BMI and DCIS in premenopausal women [12, 33-36]. The relation between BMI and postmenopausal DCIS has not been clarified yet with conflicting findings with null [11, 13, 16, 21, 34], positive [33], or inverse [37] association. A study on premenopausal women reported a stronger inverse association for in situ cancer than the association for invasive cancer $(<45)$. [12] We suppose the findings in studies of mixed premenopausal and postmenopausal women could have been influenced by the proportion of postmenopausal women among IDC and DCIS cases. Although interaction by menopausal status on the association of both IDC and DCIS with BMI was not evident in our study (Additional file 1: Table S2), the association of obesity with breast cancer needs to be further evaluated with consideration of pathologic type and menopausal status.

It has been suggested that the risk factors operating early in life such as family history might be involved in the initial stages of carcinogenesis, resulting in in situ cancer, and other factors needed to continue promoting the tumor to invasive cancer [13]. A study that found stronger association of a family history of breast cancer with DCIS than with invasive cancer, especially in younger women than in older women suggested greater genetic influence on DCIS [34]. On the other hand, the increased risk associated with the breast cancer of at least one first degree relative has been consistently similar between IDC and DCIS in many studies [21, 34, 37], suggesting an inherited predisposition to both types of breast cancer. Our study also confirmed family history of breast cancer is an important risk factor of IDC as well as DCIS. Although the association was borderline significant for DCIS, the estimate for DCIS $(\mathrm{OR}=2.14)$ was greater in strength than that for IDC $(\mathrm{OR}=1.43)$. Given that a woman with a family history of breast cancer is more likely to volunteer to health check-up, the risk of breast cancer associated with family history of breast cancer might have been underestimated in our study. Thus, the positive association of family history with DCIS and IDC in our study seems to provide strong evidence supporting the role of genetic effect on breast cancer. Although differential association with DICS versus IDC has been found for some breast cancer predisposition loci, most (76\%) of breast cancer predisposition loci previously reported for IDC were associated with DCIS in the same direction in several studies [11, 38, 39], which support strong shared genetic susceptibility of DCIS and IDC.

The risks of IDC and DCIS have been consistently found to increase in women with a history of benign breast disease $[12,16,33]$. Our study also found that benign breast disease is associated with increased risk of breast IDC as well as DCIS and the risk estimates for DCIS (OR: 2.04) were similar to that for IDC (OR: 2.31).

Regular physical activity has been proposed as an independent protective factor of breast cancer [40, 41], which we confirmed in our study. It has been scarcely evaluated whether the association with physical activity differs between IDC and DCIS. In a case-control study by Trentham-Dietz et al. [16], increasing frequency of physical activity in early adulthood was inversely associated with the risk of invasive cancer (OR $(95 \% \mathrm{CI})$ per frequency/week: $0.96(0.93,0.99))$, and it did not significantly differ from the association between in situ cancer and physical activity (OR(95\% CI): 0.99(0.92,1.07)). We also found that regular physical activity was inversely associated with the risk of IDC as well as DCIS and the estimates were not different each other. Studies have consistently reported that alcohol consumption had a positive association with breast cancer [42, 43]. In the present study, we found that DCIS have a stronger association with alcohol consumption than IDC had, although the difference was not statistically different $(P=0.105)$. Trentham-Dietz et al. also reported that ORs (95\% CI) for $\geq 183 \mathrm{~g} /$ week of alcohol intake were $2.34(1.32,4.16)$ for DCIS and $1.76(1.37,2.25)$ for IDC 
but without statistical difference between them [16], which is very consistent with the findings of our study.

The present study had some limitations. First, our study may have weakness with respect to representativeness that is commonly innate in a hospital-based case-control study. As controls were recruited from participants in a health checkup program, selection bias may exist. We tried to overcome this bias by considering a wide range of covariates including health behaviors such as physical exercise, alcohol consumption, and smoking habit. Second, we recruited cases in two ways (retrospectively or prospectively), and this might have incurred bias in the study findings due to the health behavior modification or recall bias. To reduce the probable confounding by the heterogeneous recruitment method, we adjusted for the recruitment method. However, the significant interaction by the recruitment method on the association of IDC with physical exercise and ever-use of estrogen replacement with different direction of the estimates for the association between the retrospectively (positive) recruited subjects and prospectively (inverse) recruited subjects suggests that careful interpretation is necessary, especially for the association of IDC and those two factors. Third, we could not consider the mode of breast cancer detection and could not examine the association of age at menopause, age at first birth, lactation, and oral pill with IDC and DCIS because of the lack of information. Fourth, because of the low proportion of DCIS among breast cancer, we may have included relatively small number of DCIS cases in this study and, thus study power could have been inadequate. Finally, given that 'benign breast disease' constitutes a heterogeneous group of breast lesions, evaluation of breast cancer risk associated with the benign breast disease could have been too vague to give useful clinical information.

On other hand, our study has some strength. First, the influence of age and menopausal status was strictly controlled through individual case-control matching and statistical adjustment. Second, a wide range of covariates was considered: BMI, age at menarche, number of children, use of estrogen replacement therapy, lifestyle factors, previous benign breast disease, and family history of breast cancer among first-degree relatives. Third, we measured MD quantitatively using a computerassisted thresholding technique.

\section{Conclusions}

In conclusion, differential associations of DCIS with mammographic density and risk factors as compared with the associations of IDC were not evident. This finding suggests that IDC and DCIS develop through the shared causal pathways.

\section{Additional file}

Additional file 1: Table S1. Influence of the method of subjects recruitment on the association of the clinical, reproductive, and mammographic density characteristics with invasive ductal carcinoma and ductal carcinoma in situ. Table S2 Association of body mass index with breast cancer according to menopausal status. (DOCX 23 kb)

\section{Abbreviations \\ ADA: absolute mammographic dense area; DCIS: ductal carcinoma in situ; ER: estrogen receptor; HER2: Cerb2 receptor; IDC: invasive ductal cancer; MD: mammographic density; PDA: percent mammographic dense area; PgR: progesterone receptor}

\section{Funding}

This research was supported by the Basic Science Research Program through the National Research Foundation of Korea (NRF) funded by the Ministry of Science, ICT, and Future Planning (2014R1A2A2A01002705). The funding sources had no involvement in conducting this study.

Availability of data and materials

Data sharing is not available because study participants did not provide consent to data sharing.

\section{Authors' contributions}

HK, JS contributed to building the conception and design of the work, analyzing and interpretation of data, and drafting and revision of the article. JEL and SJN contributed to building the conception, data collection, interpretation of data, and revising the article. TLN, JLH contributed to building the conception, design of the work, interpretation of data, and revising the article. Y-MS (Corresponding author) contributed to building the conception and design of the work, data collection, clarifying important intellectual content of study finding, and critical revision from draft version to final version of the article. All authors participated in final approval of the version to be published and contributed to ensuing that questions related to the accuracy or integrity of any part of the work are appropriately investigated and resolved.

Ethics approval and consent to participate

This study was approved by the Institutional Review Board of Samsung Medical Center (SMC2011-06-052). The Board waived informed consent for the retrospectively recruited subjects and all prospectively recruited subjects provided written informed consent.

Consent for publication

Not applicable

Competing interests

The authors declare that they have no competing interests.

\section{Publisher's Note}

Springer Nature remains neutral with regard to jurisdictional claims in published maps and institutional affiliations.

\section{Author details}

${ }^{1}$ Department of Family Medicine, Samsung Medical Center, Sungkyunkwan University School of Medicine, 81 Irwon-ro, Gangnam-gu, Seoul 135-710, South Korea. ${ }^{2}$ Department of Surgery, Samsung Medical Center, Sungkyunkwan University School of Medicine, Seoul, South Korea. ${ }^{3}$ Melbourne School of Population and Global Health, Centre for Epidemiology and Biostatistics, University of Melbourne, Carlton, VIC, Australia. ${ }^{4}$ Department of Epidemiology, School of Public Health and Environment, Seoul National University, Seoul, South Korea. ${ }^{5}$ Department of Family Medicine, Konkuk University Medical Center, Konkuk University School of Medicine, Seoul, South Korea. 
Received: 5 January 2017 Accepted: 24 November 2017

Published online: 05 December 2017

\section{References}

1. Levi F, Te VC, Randimbison L, La Vecchia C. Trends of in situ carcinoma of the breast in Vaud, Switzerland. Eur J Cancer. 1997;33:903-6.

2. Li Cl, Daling JR. Changes in breast cancer incidence rates in the United States by histologic subtype and race/ethnicity, 1995 to 2004. Cancer Epidemiol Biomark Prev. 2007;16:2773-80.

3. Min SY, Kim Z, Hur MH, Yoon CS, Park EH, Jung KW, et al. The basic facts of Korean breast cancer in 2013: results of a Nationwide survey and breast cancer registry database. J Breast Cancer. 2016;19:1-7.

4. Burstein HJ, Polyak K, Wong JS, Lester SC, Kaelin CM. Ductal carcinoma in situ of the breast. N Engl J Med. 2004;350:1430-41.

5. Arvold ND, Punglia RS, Hughes ME, Jiang W, Edge SB, Javid SH, et al. Pathologic characteristics of second breast cancers after breast conservation for ductal carcinoma in situ. Cancer. 2012:118:6022-30.

6. Levi F, Randimbison L, Te VC, La Vecchia C. Invasive breast cancer following ductal and lobular carcinoma in situ of the breast. Int J Cancer. 2005;116:820-3.

7. Collins LC, Tamimi RM, Baer HJ, Connolly JL, Colditz GA, Schnitt SJ. Outcome of patients with ductal carcinoma in situ untreated after diagnostic biopsy: results from the Nurses' health study. Cancer. 2005;103:1778-84.

8. Zagouri F, Sergentanis TN, Zografos GC. Precursors and preinvasive lesions of the breast: the role of molecular prognostic markers in the diagnostic and therapeutic dilemma. World J Surg Oncol. 2007:5:57.

9. Mommers EC, Leonhart AM, Falix F, Michalides R, Meijer CJ, Baak JP, et al. Similarity in expression of cell cycle proteins between in situ and invasive ductal breast lesions of same differentiation grade. J Pathol. 2001;194:327-33.

10. Holzman D. Scientists show that invasive breast cancer develops from early cancer cells. J Natl Cancer Inst. 1995:87:710-1.

11. Reeves GK, Pirie K, Green J, Bull D, Beral V. Million women study C. Comparison of the effects of genetic and environmental risk factors on in situ and invasive ductal breast cancer. Int J Cancer. 2012;131:930-7.

12. Weiss HA, Brinton LA, Brogan D, Coates RJ, Gammon MD, Malone KE, et al. Epidemiology of in situ and invasive breast cancer in women aged under 45. Br J Cancer. 1996;73:1298-305.

13. Brinton LA, Hoover R, Fraumeni JF Jr. Epidemiology of minimal breast cancer. JAMA. 1983;249:483-7.

14. Lambe M, Hsieh CC, Tsaih SW, Ekbom A, Trichopoulos D, Adami HO. Parity, age at first birth and the risk of carcinoma in situ of the breast. Int J Cancer. 1998;77:330-2.

15. Dubin N, Hutter RV, Strax P, Fazzini EP, Schinella RA, Batang ES, et al. Epidemiology of minimal breast cancer among women screened in new York City. J Natl Cancer Inst. 1984;73:1273-9.

16. Trentham-Dietz A, Newcomb PA, Storer BE, Remington PL. Risk factors for carcinoma in situ of the breast. Cancer Epidemiol Biomark Prev. 2000;9:697-703.

17. Boyd NF, Martin $\amalg$, Yaffe MJ, Minkin S. Mammographic density and breast cancer risk: current understanding and future prospects. Breast Cancer Res. 2011;13:223.

18. Kim BK, Choi YH, Nguyen TL, Nam SJ, Lee JE, Hopper JL, et al. Mammographic density and risk of breast cancer in Korean women. Eur J Cancer Prev. 2015:24: 422-9.

19. Yaghjyan L, Colditz GA, Collins LC, Schnitt SJ, Rosner B, Vachon C, et al. Mammographic breast density and subsequent risk of breast cancer in postmenopausal women according to tumor characteristics. J Natl Cancer Inst. 2011;103:1179-89.

20. Gill JK, Maskarinec G, Pagano I, Kolonel LN. The association of mammographic density with ductal carcinoma in situ of the breast: the multiethnic cohort. Breast Cancer Res. 2006:8:R30.

21. Reinier KS, Vacek PM, Geller BM. Risk factors for breast carcinoma in situ versus invasive breast cancer in a prospective study of pre- and postmenopausal women. Breast Cancer Res Treat. 2007;103:343-8.

22. Pollan M, Ascunce N, Ederra M, Murillo A, Erdozain N, Ales-Martinez J, et al. Mammographic density and risk of breast cancer according to tumor characteristics and mode of detection: a Spanish population-based casecontrol study. Breast Cancer Res. 2013:15:R9.

23. Sala E, Solomon L, Warren R, McCann J, Duffy S, Luben R, et al. Size, node status and grade of breast tumours: association with mammographic parenchymal patterns. Eur Radiol. 2000;10:157-61.
24. Ursin G, Hovanessian-Larsen L, Parisky YR, Pike MC, Greatly WAH. Increased occurrence of breast cancers in areas of mammographically dense tissue. Breast Cancer Res. 2005;7:R605-8

25. Boyd NF, Jensen HM, Cooke G, Han HL. Relationship between mammographic and histological risk factors for breast cancer. J Natl Cancer Inst. 1992;84:1170-9.

26. Ernster VL, Ballard-Barbash R, Barlow WE, Zheng Y, Weaver DL, Cutter G, et al. Detection of ductal carcinoma in situ in women undergoing screening mammography. J Natl Cancer Inst. 2002;94:1546-54.

27. Ghosh K, Brandt KR, Sellers TA, Reynolds C, Scott CG, Maloney SD, et al. Association of mammographic density with the pathology of subsequent breast cancer among postmenopausal women. Cancer Epidemiol Biomark Prev. 2008;17:872-9.

28. Bertrand KA, Tamimi RM, Scott CG, Jensen MR, Pankratz V, Visscher D, et al. Mammographic density and risk of breast cancer by age and tumor characteristics. Breast Cancer Res. 2013:15:R104.

29. Maskarinec G, Meng LA. Case-control study of mammographic densities in Hawaii. Breast Cancer Res Treat. 2000;63:153-61.

30. Ursin G, Ma H, AH W, Bernstein L, Salane M, Parisky YR, et al. Mammographic density and breast cancer in three ethnic groups. Cancer Epidemiol Biomark Prev. 2003:12:332-8.

31. Nagao $Y$, Kawaguchi $Y$, Sugiyama Y, Saji S, Kashiki Y. Relationship between mammographic density and the risk of breast cancer in Japanese women: a case-control study. Breast Cancer. 2003;10:228-33.

32. McCormack VA. Dos Santos Silva I. Breast density and parenchymal patterns as markers of breast cancer risk: a meta-analysis. Cancer Epidemiol Biomark Prev. 2006:15:1159-69.

33. Longnecker MP, Bernstein L, Paganini-Hill A, Enger SM, Ross RK. Risk factors for in situ breast cancer. Cancer Epidemiol Biomark Prev. 1996:5:961-5.

34. Kerlikowske K, Barclay J, Grady D, Sickles EA, Ernster V. Comparison of risk factors for ductal carcinoma in situ and invasive breast cancer. J Natl Cancer Inst. 1997:89:76-82.

35. Patel AV, Press MF, Meeske K, Calle EE, Bernstein L. Lifetime recreational exercise activity and risk of breast carcinoma in situ. Cancer. 2003:98:2161-9.

36. Swanson CA, Coates RJ, Schoenberg JB, Malone KE, Gammon MD, Stanford $J$, et al. Body size and breast cancer risk among women under age 45 years. Am J Epidemiol. 1996;143:698-706.

37. Meeske K, Press M, Patel A, Bernstein L. Impact of reproductive factors and lactation on breast carcinoma in situ risk. Int J Cancer. 2004;110:102-9.

38. Petridis C, Brook MN, Shah V, Kohut K, Gorman P, Caneppele M, et al. Genetic predisposition to ductal carcinoma in situ of the breast. Breast Cancer Res. 2016;18:22

39. Michailidou K, Hall P, Gonzalez-Neira A, Ghoussaini M, Dennis J, Milne RL, et al. Large-scale genotyping identifies 41 new loci associated with breast cancer risk. Nat Genet. 2013:45:353-61.61e1-2

40. Monninkhof EM, Elias SG, Vlems FA, van der Tweel I, Schuit AJ, Voskuil DW, et al. Physical activity and breast cancer: a systematic review. Epidemiology. 2007;18:137-57

41. Yang D, Bernstein L, Physical WAH. Activity and breast cancer risk among Asian-American women in Los Angeles: a case-control study. Cancer. 2003; 97:2565-75

42. Smith-Warner SA, Spiegelman D, Yaun SS, van den Brandt PA, Folsom AR, Goldbohm RA, et al. Alcohol and breast cancer in women: a pooled analysis of cohort studies. JAMA 1998:279:535-40.

43. Tjonneland A, Christensen J, Olsen A, Stripp C, Thomsen BL, Overvad K, et al. Alcohol intake and breast cancer risk: the European prospective investigation into cancer and nutrition (EPIC). Cancer Causes Control. 2007; 18:361-73. 